\title{
Pour une valorisation de la diversité sexuelle dans la salle de classe : de l'hétéronormativité au conflit queer
}

\author{
Hasheem Hakeem, Université Simon Fraser \\ Lauréat du Prix APFUCC 2018 de la meilleure communication par un.e jeune chercheur.e
}

Comme l'a expliqué Michel Foucault dans Surveiller et punir (1975), la société disciplinaire qui émerge au cours du dix-neuvième siècle transforme les relations de pouvoir. Nous passons d'un pouvoir centré sur la mort, déployé par le souverain de manière très visuelle et théâtrale, vers un pouvoir relationnel, donc un pouvoir centré sur l'administration de la vie. Ce nouveau type de pouvoir fonctionne à travers des appareils disciplinaires qui ont pour but de classer et de hiérarchiser les individus en fonction d'une construction sociale et politique qui impose des normes, des lois et des règles. Dans la société disciplinaire, le pouvoir est l'effet des relations entre individus qui produisent ce que Foucault appelle des disciplines : «La discipline n'est plus simplement un art de répartir des corps, d'en extraire et d'en cumuler du temps, mais de composer des forces pour obtenir un appareil efficace » (192). Pour Foucault, toute institution, que ce soit l'école, l'hôpital ou la prison, est un appareil disciplinaire qui sert à conditionner un individu, à le définir, à lui imposer une identité et finalement à le faire fonctionner à partir de cette identité. Ainsi, la discipline fabrique des corps soumis et dociles « [...] non pas simplement pour qu'ils fassent ce qu'on désire, mais pour qu'ils opèrent comme on veut [...]» (162).

Si nous suivons Foucault, l'identité ne préexiste pas au pouvoir des institutions disciplinaires qui la définit, la détermine et la produit. Cette manière de former le corps à partir d'institutions disciplinaires est ancrée dans un processus de normalisation : «En somme, l'art de punir, dans le régime du pouvoir disciplinaire, ne vise ni l'expiation, ni même exactement la répression [...]. La pénalité perpétuelle qui traverse tous les points, et contrôle tous les instants des institutions disciplinaires compare, différencie, hiérarchise, homogénéise, exclut. En un mot elle normalise » (214-215). Comme l'individu n'existe pas avant que l'appareil disciplinaire ne le produise, il est entièrement soumis à la norme qui le produit, ce qui limite la myriade de possibilités de cheminement identitaire. Foucault utilise la métaphore du panoptique pour illustrer l'effet de l'emprise des appareils disciplinaires qui entraînent chez l'individu « [...] un état conscient et permanent de visibilité qui assure le fonctionnement automatique du pouvoir » (234). Foucault 
nous dit que le panoptique, un système de surveillance qui assure la discipline de l'individu en fonction des normes dominantes de la société dont il fait partie, représente la nouvelle structure des institutions disciplinaires. Mais à force d'être constamment surveillé, l'individu intériorise le pouvoir. Autrement dit, l'institution disciplinaire, d'emblée un mécanisme de surveillance qui sert à dresser et à normaliser l'individu, est transformée en système d'autosurveillance et, donc, « [au] milieu de tous ces dispositifs de normalisation qui se resserrent, la spécificité de la prison et son rôle de joint perdent de leur raison d'être » (358). L'individu passe d'un sujet discipliné à un sujet auto-discipliné, n'ayant plus besoin de la prison, voire de l'institution disciplinaire pour le normaliser parce qu'il se normalise en intériorisant et en performant les normes des appareils disciplinaires.

Dans Histoire de la sexualité I : La volonté de savoir (1976), Foucault précise que la discipline de l'individu sert finalement à réguler de grandes populations de millions de personnes, voire à classer et à hiérarchiser un ensemble d'individus dans une population en fonction de leur identité disciplinée. Ce pouvoir qui contrôle la vie au niveau de la discipline de l'individu et au niveau de la régulation de la population, c'est ce que Foucault appelle la biopolitique. Pour lui, c'est dans le domaine de la sexualité que se rencontrent ces deux pôles : «Le sexe est accès à la fois à la vie du corps et à la vie de l'espèce. On se sert de lui comme matrice des disciplines et comme principe des régulations » (192). Dans ce contexte, l'identité sexuelle est un mécanisme essentiel pour le fonctionnement du système de pouvoir biopolitique. Il faut identifier ceux qui méritent de vivre (les humains qui se plient et se conforment aux normes) et ceux qu'il est nécessaire d'éliminer (les humains qui résistent, qui sont incapables d'intégrer les normes, qui échouent à suivre la norme ou encore qui transgressent et subvertissent ces limites et ces systèmes normatifs).

À partir de ces conceptions de Foucault, nous pouvons considérer que l'école est un système de pouvoir normalisateur au service d'une logique de contrôle social identitaire qui a pour but de produire des sujets hétéronormés et binaires. En fait, dans Constructing Sexualities and Gendered Bodies in School Spaces (2017), Jón Ingvar Kjaran affirme que l’école, que ce soit dans le contexte canadien, américain, anglais ou nordique, demeure largement un lieu hétéronormatif : «In most schools, the curriculum is rather heteronormative and any lessons or education about LGBTQ people and reality is lacking or non-existent. This is in accordance with qualitative research from the UK, USA and Canada, which has indicated that students in general rarely receive 
any education on sexuality, LGBTQ reality and issues, or gender at school » (39). Par conséquent, si la seule parole qui a le droit de circuler dans l'école est toujours liée à l'hétéronormativité, les élèves queer font face, de manière plus générale, à un pouvoir biopolitique disciplinaire et régulateur et, de manière plus particulière, aux mécanismes de contrôle du patriarcat, de l'hétéronormativité et du binarisme de genre. Dans le contexte de ce système de pouvoir que nous appelons «l'école », comment prendre la parole, comment faire valoir la diversité sexuelle et de genre, comment avoir le courage de la vérité et comment avoir la possibilité de tout dire ? De plus, quelles sont les conditions nécessaires à la liberté de parole dans l'école afin que les voix queer puissent s'élever, être entendues et surtout, être comprises ? Cet article propose de répondre à ces questions en prêtant attention à la manière dont une pédagogie queer peut réussir à transgresser l'hétéronormativité et à faire place à une plus grande diversité sexuelle, diversité d'identité de genre et diversité d'expression de genre.

\section{Productivité du conflit dans la salle de classe}

Pour répondre à ces problématiques, la réflexion que Chantal Mouffe a proposée sur le conflit dans L'illusion du consensus (2016), nous sera particulièrement utile. Mouffe explique que la structure libérale de la/nos démocratie(s), aussi contradictoire que cela puisse paraître, est à l'origine des antagonismes dans notre/nos société(s) parce que, lorsque nous « [...] ne faisons pas face à des options clairement différenciées parmi lesquelles trancher» (11), nous voulons naturellement résister à cette hégémonie existante qui ne reconnaît pas notre voix en tant que citoyen. Dans une société de consensus, comme l'écrit Mouffe, il n'existe pas de réelle acceptation de la pluralité parce que la société est conçue comme un «nous » homogène opérant sous la forme d'une illusion de consensus (9). Niant la dimension conflictuelle de la vie sociale, une société de consensus nous permet de vivre ensemble en faisant semblant d'ignorer nos différences, c'est-àdire en les cachant, en les masquant et en les censurant. C'est précisément la raison pour laquelle il y a une grande tension entre le fait, d'une part, de s'engager à faire entrer dans la salle de classe une diversité d'identités sexuelles et genrées et, d'autre part, les risques, les dangers, les obstacles, les peurs de le faire, parce que nous savons que nous serons confrontés à toutes sortes de réactions alimentées par de nombreux préjugés renforcés par l'illusion du consensus qu'il est interdit de briser. Ainsi, le consensus s'inscrit dans la logique patriarcale, hétéronormative et binaire dans la mesure où il sert à normaliser, uniformiser et hiérarchiser les individus et les populations. 
Pour Mouffe, que nous le voulons ou pas, nous vivons dans des sociétés hyper pluralistes, fragmentées et hétérogènes et, donc, le conflit est une réalité inévitable qu'il faut pouvoir affronter efficacement. Afin de faire valoir la diversité sexuelle, la diversité d'identité de genre et la diversité d'expression de genre dans la salle de classe, il faut plutôt tenter de passer à une légitimation du conflit (180) qui brisera le consensus. Si le consensus maintient le statu quo hétéronormatif et prévoit de manière uniforme la sexualité et le genre des élèves, le conflit, quant à lui, bouleverse ce statu quo en le remettant en question par le biais d'une pédagogie queer. Cette pédagogie queer valorise le très grand spectre des potentialités des modes de vie, des pratiques, des relations et de la sexualité de l'être humain. En soulignant l'immense diversité sexuelle qui existe chez tous les êtres humains et en rejetant la présomption d'hétérosexualité, la pédagogie queer déclenche l'expérience du conflit dans la salle de classe, ce qui donne aux élèves des outils pour être des citoyens conscients et actifs dans une société démocratique qui défend et valorise la pluralité, la différence, l'hétérogénéité et la fragmentation. Le conflit permet donc aux élèves de prendre conscience de leur propre diversité sexuelle et de celle de leurs pairs, alors que le consensus dissimule cette diversité derrière une définition homogène du corps, de la sexualité, du genre et de l'affect, qui ne va pas de soi. Pour ces raisons, le conflit est une condition nécessaire pour, non seulement libérer la parole queer, mais pour atteindre la justice sociale au sein des institutions scolaires.

Notre analyse portera dans un premier temps sur L'enfant mascara (2016) de l'écrivain québécois Simon Boulerice, roman qui lie la réalité du meurtre d'une élève transgenre à la réflexion queer sur ce meurtre à travers la fiction, et dans un deuxième temps sur l'étude de la revue canadienne queer kwiR publiée par des étudiants de l'école secondaire.

\section{La force queer dans L'enfant mascara de Simon Boulerice}

Écrit sous la forme d'un journal intime, L'enfant mascara raconte l'expérience de Larry/Leticia, une fille transgenre âgée de quinze ans qui est tuée par balles par Brandon McInerney après qu'elle lui demande d'être son valentin. Le roman est composé d'une série d'entrées de journal qui nous plongent dans le quotidien à la fois banal et éclectique de Leticia : sa vie pauvre, son amour pour Joan Crawford, son père agressif, son arrogance, son désir de se maquiller, ainsi que toutes ses imperfections. Sur un plan pédagogique, la focalisation sur un personnage dont l'identité sexuelle et genrée n'est pas conforme à l'hétérosexualité ou au 
binarisme de genre, contribue à remettre en question le consensus du tabou et de l'interdit autour de la diversité sexuelle, de la diversité d'identité de genre et de la diversité d'expression de genre dans la salle de classe. De surcroît, l'histoire de Leticia est un outil pour déclencher une réflexion ; elle cristallise une idée qui commence dans la salle de classe, mais dont le développement n'est pas limité à un espace fermé et immuable. Le simple acte de lire L'enfant mascara nous permet d'entrer dans le monde d'une élève transgenre qui, grâce à ses imperfections, assume une vie authentique libre des fausses apparences de l'hétéronormativité. Nous pouvons ainsi inscrire la littératie comme pratique sociale qui engage le rapport au monde; elle crée ce que Michael Warner appelle des «counterpublics» ou des espaces discursifs où peuvent être remises en question les normes dominantes : «Counterpublics are spaces of circulation in which it is hoped that the poesis of scene making will be transformative, not replicative merely » (Publics, 122 ; je souligne). Étant donné que Leticia représente tout ce qui est défini comme étant hors norme et donc anormal par le système scolaire, le fait de privilégier à l'intérieur de ce même espace son histoire et de la rendre plus visible peut contribuer à défier et à transformer la construction hétéropatriarcale de la salle de classe.

L'importance de ce roman, d'un point de vue pédagogique, est qu'il défie les normes dominantes du genre et de la sexualité sur lesquelles est fondé le consensus et, donc, il déclenche un conflit. L'enfant mascara est avant tout un roman centré sur la vie, les pensées, les désirs d'une personne transgenre. Bien que Leticia soit tuée par Brandon et que ce meurtre soit annoncé dès le début du roman, l'auteur choisit de clore sur la voix de Leticia qui prime, même après sa mort, ce qui renforce une sorte de tribune permanente qui perdure avec le temps et reste inaltérable. Ayant exploité ce dernier chapitre intitulé Mirador dans ma salle de classe, j'ai constaté qu'il est un exemple pertinent d'un contre-discours à la perspective hétéronormative qui tente d'effacer et de rendre invisible la multiplicité des identités et des expériences queer. Bien que nous puissions interpréter la mort de Leticia comme une conclusion logique qui s'inscrit dans le consensus de la censure de la diversité sexuelle, Boulerice semble faire exister cette diversité en offrant à Leticia la seule tribune et en insistant sur sa force, malgré sa mort tragique :

Un jour, il fera frais, mais beau, et tu sortiras de prison. Tu auras trente-neuf ans, mais peut-être aussi un peu moins. Ce sera une journée plus venteuse. Tu enfileras ton anorak rouge, plus par habitude que par nécessité. Une étrange brise s'infiltrera sous la toile et soulèvera ton léger manteau, comme un pneumatique que l'on gonfle à la pompe. Tu croiras à une présence magique - à un ange gardien ? - tu auras 
raison. Tu comprendras que c'est moi qui veille sur toi, pour ta nouvelle vie qui s'entamera alors. (180)

À la fin du roman, Leticia devient un ange gardien pour Brandon ; même le fait de la tuer ne peut guère faire disparaître la force qu'elle incarne. En nous laissant avec l'image de Leticia veillant sur Brandon après sa sortie de prison, Boulerice éternise symboliquement la subjectivité queer. Étant donné que Boulerice décrit en toute sincérité et authenticité la tragédie de la mort d'une personne transgenre et les réactions qui en découlent, ce dernier chapitre est d'autant plus inattendu parce qu'il se termine sur la voix de Leticia. Son agentivité queer semble donc atteindre son paroxysme par le biais de la mort, suggérant que le queer échappe à toute tentative de néantisation par le système hétéronormatif et patriarcal.

Par ailleurs, dans une interprétation visuelle de ce chapitre, un de mes élèves montre à quel point Leticia, remplie de couleurs vibrantes et coiffée à la Joan Crawford, prend toute la place, contrairement à Brandon qui, lui, demeure petit et sombre, relégué à l'arrière-plan sociétal. Brandon, figuré dans cette image comme métaphore de l'hétéronormativité, est réduit à l'invisibilité, alors que Leticia, qui représente le queer, domine tout l'espace. L'art que produit cet élève et pour qui le roman a été un tremplin réflexif, devient ici une stratégie pour libérer la parole queer en augmentant la visibilité des identités LGBTQ2+ dans l'imaginaire collectif de l'école et des élèves. La représentation visuelle de la puissance de Leticia va à l'encontre du consensus en affirmant que le queer est de l'ordre de l'incontrôlé et de l'incontrôlable ; il ne peut être ni normalisé ni régulé par la société hétéronormative et patriarcale qui se retrouve affaiblie et réduite à peau de chagrin par cette force queer. Si Brandon ressent le besoin de tuer Leticia pour prouver son hétérosexualité et sa masculinité, tout ce qu'il finit par nous montrer, c'est son extrême inconfort et sa fragilité par rapport à son identité hétéronormée. Ainsi, l'exiguiité et la noirceur pour représenter Brandon dans la peinture mentionnée montre symboliquement à quel point le système hétéronormatif est fragile parce qu'il s'effondre face à l'instabilité et à la capacité transformatrice du queer. D'un point de vue pédagogique, l'analyse du chapitre Mirador, avec son interprétation visuelle, nous permet de comprendre la manière dont la parole queer est libérée grâce à une fragilisation du consensus hétéronormatif.

Notons également que cette peinture a été offerte à Boulerice en cadeau devant les élèves à la fin d'une conférence que ce dernier donnait dans l'école. L'acte de faire rentrer une peinture queer dans un espace qui privilégie la parole et le geste hétéronormés contribue à queeriser 
l'espace scolaire, voire à créer des points de tension et de résistance aux discours hétéronormatifs dans l'école. Selon Warner, « [to] seek out queer culture, to interact with it and learn from it, is a kind of public activity. It is a way of transforming oneself, and at the same time helping to elaborate a commonly accessible world » (Trouble, 71$)$.

Aller vers des textes comme L'enfant mascara, qui valorisent l'expérience queer, est une véritable ouverture vers un monde plus accessible, un monde caractérisé par la transformation et par le changement, et non par l'immobilisme des normes et des pratiques sexuelles. Par le biais de l'art et de la lecture, l'élève déplace l'invisibilité queer vers l'affirmation de la création, s'appropriant l'espace scolaire hétéronormatif pour ainsi le transformer en lieu de construction de sa propre agentivité et de sa subjectivité queer.

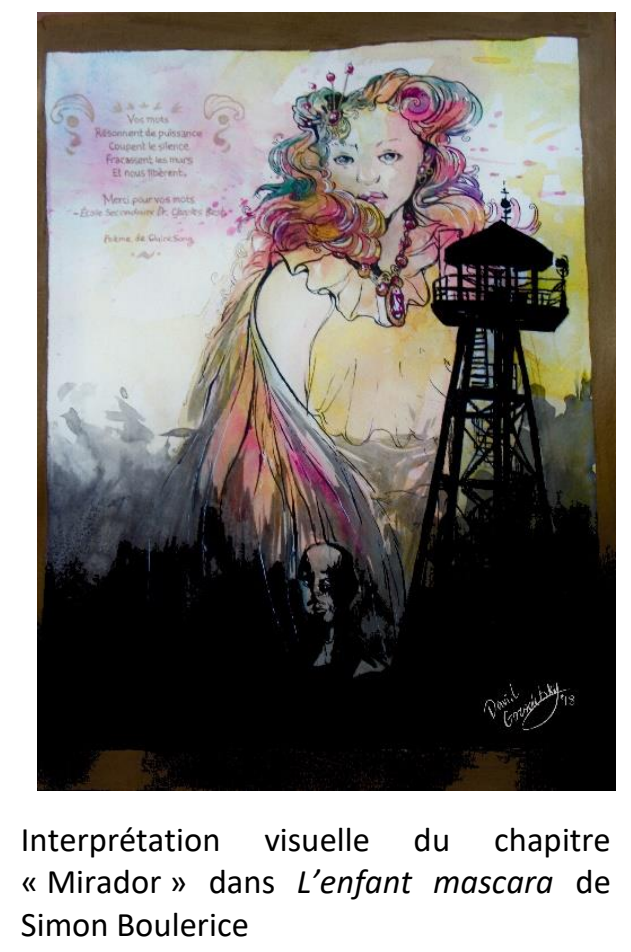

\section{kwiR, ou comment s'attaquer à l'hétéronormativité ?}

Un autre exemple de la productivité socio-politique du conflit est la revue $k w i R^{1}$, qui a été fondée en 2017 dans le cadre de la Journée Internationale contre l'homophobie, la transphobie et la biphobie par un groupe d'élèves inscrits à mon cours de Journalisme 11/12 à l'école secondaire Dr. Charles Best à Coquitlam en Colombie-Britannique. Cette revue, comme en témoigne le soustitre Pour en finir avec l'hétéronormativité, est composée d'un ensemble d'éditoriaux, de poèmes et de caricatures qui ont pour but de valoriser la diversité sexuelle et d'émanciper les élèves et les 
familles LGBTQ2+ qui souffrent en silence et dont la sexualité et l'identité de genre sont activement marginalisées par les pratiques hétéronormatives de l'école. Si nous prenons l'exemple de la page couverture du premier numéro de la revue, paru en mai 2018, nous constatons qu'il s'agit de deux individus, dont le genre n'est pas défini, qui s'embrassent. Cette première image, couplée avec le message «d'en finir avec l'hétéronormativité », est à la fois puissante et perturbante parce qu'elle met de l'avant de manière conflictuelle et inattendue l'existence du queer dans un espace où celui-ci est normalement invisible. En voulant représenter des pratiques, des modes de vie et des manières de réfléchir autres que celles institutionnalisées par la société hétéronormative et patriarcale, la revue annonce dès la page couverture son opposition véhémente au consensus mis en place par l'institution scolaire.

Cette revue, à part le fait qu'elle rend plus visible la diversité sexuelle, la diversité d'identité de genre et la diversité d'expression de genre, est à la fois un outil important pour développer la pensée critique des élèves. Selon Kjaran, « [...] they [les élèves] need to develop critical literacy and cultural awareness in order to be critical toward their environment, and in my view, it is the role of teachers to encourage them to do so » (181-182). En d'autres mots, la pensée critique pourrait être considérée comme une forme de conflit appelant les élèves à se questionner, à se redéfinir et à se « déplacer » constamment en fonction des discours dominants sur le genre et la sexualité. Une pédagogie qui n'entre pas en conflit avec l'hétéronormativité ne peut donc pas amener les élèves à entamer une pensée critique, car, en évitant le conflit, elle les socialise dans un curriculum qui renforce la présupposition d'hétérosexualité universelle et omniprésente. Le conflit serait donc une condition indispensable au développement de la pensée critique au sein des institutions scolaires. Si nous prenons l'exemple du binarisme de genre comme construction sociale et politique moderne (le sujet de l'un des articles de la revue $k w i R$ ), cet article crée un conflit par rapport au discours hétéronormatif qui suppose que le genre soit naturellement binaire. Ce conflit serait éthiquement nécessaire pour déplacer un discours idéologique sur le genre qui ne correspond pas à la réalité concrète de la diversité d'identité de genre et de la diversité d'expression de genre au sein de laquelle se déroule l'existence quotidienne des jeunes élèves. Il en est de même pour d'autres sujets abordés dans la revue, tels que la construction de la masculinité hétéropatriarcale et la pseudoscience de la thérapie de conversion. Pour ces raisons, à travers le conflit, les élèves apprennent à poser des questions critiques et à être conscients des différents discours qui les influencent et les construisent (Kjaran 182), alors que le consensus naturalise le 
discours hétéronormatif en projetant dans le curriculum et dans la culture scolaire une illusion de l'hétérosexualité comme la seule réalité possible.

Passons par la suite à l'analyse d'une image dans la revue numéro 1 en mai 2018. La quatrième de couverture caricature un enfant qui tente de sortir du placard, alors que son enseignant y est adossé en lisant à ses élèves. La caricature insiste sur le rôle de l'institution scolaire dans la préservation du placard et, donc, de l'invisibilité LGBTQ2+ au sein des établissements scolaires. Bien que l'enseignant disparaisse, ce sont les enfants qui prennent la relève et assument le rôle d'agents de cette matrice hétérosexuelle, de l'hétéronormativité et du patriarcat, qui ont pour but de réguler et de contrôler la sexualité dans toutes les institutions de la vie sociale. Le placard, comme l'explique Eve Sedgwick dans Epistemology of the Closet (1999), est produit par une logique binaire selon laquelle l'hétérosexualité doit avoir une liberté totale, alors que le queer doit être emprisonné dans des limites très restreintes. Comme la société hétéronormative valorise uniquement l'hétérosexualité, elle impose dans l'espace public la logique hétérosexuelle et produit des placards dans lesquels sont enfermées des personnes queer afin de supprimer et de rendre invisible leur diversité sexuelle. La juxtaposition du noir et du blanc renforce davantage cette dichotomie entre l'hypervisibilité hétéronormative et l'invisibilité queer. Dans le contexte de la caricature, le placard devient une prison psychique parce que les enfants queer doivent être invisibles et cette invisibilité est renforcée par l'institution scolaire qui interdira que l'on soit conscient de manière explicite de la diversité sexuelle, comme le démontre les yeux bandés des enfants. C'est précisément la raison pour laquelle « [the] closet is the defining structure for gay oppression in this century » (71) parce que le placard efface la subjectivité et l'agentivité queer, d'où l'importance de réinventer et de redéfinir des pratiques et des modes de vie qui contribueront à détruire le placard. Ainsi, l'exemple de l'ambiguïté sexuelle représentée par la page couverture de la revue brouille la frontière normative entre homme/femme, entre hétérosexuel/homosexuel, en déconstruisant dans l'espace scolaire les binarismes qui renforcent l'hétéronormativité. 


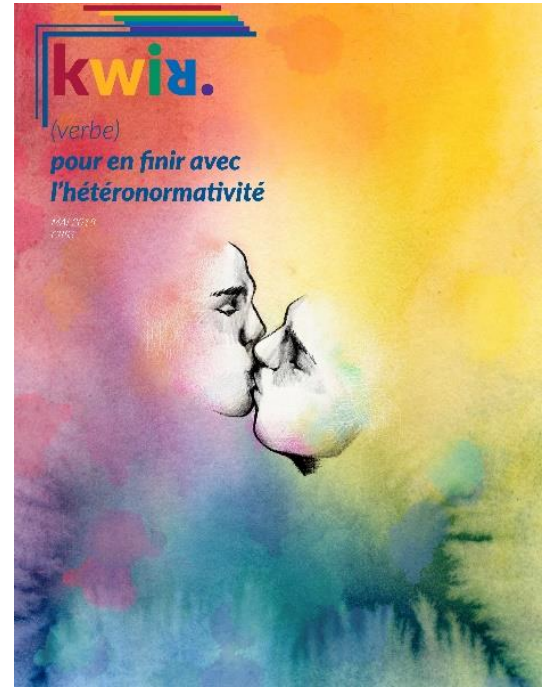

Page couverture de la revue $k w i R$

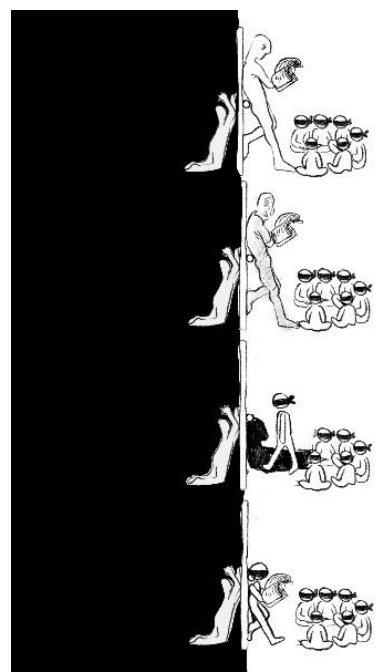

Quatrième de couverture de la revue kwiR

Il convient enfin de constater que cette caricature était illustrée et que le concept a été développé par un élève queer. Puisque la caricature était utilisée dans mon cours de français pour entamer une discussion critique, l'expérience du placard a pu être abordée dans la salle de classe dans une perspective queer. Bien que la caricature déconcerte, cet inconfort permet aux élèves de prendre conscience de l'inconfort suscité par l'hétéronormativité. À travers des analyses et des discussions, les élèves constatent que le placard est une construction hétéronormative qui discipline les enfants à confiner le queer dans la noirceur et dans l'ombre, tout en privilégiant aveuglement l'identité hétérosexuelle. En fait, lors d'une analyse faite en classe, un élève avait remarqué que l'adulte dans l'image était le seul personnage qui n'avait pas les yeux bandés, débouchant sur la conclusion que le queer est consciemment anéanti par les institutions scolaires, alors que l'ignorance des enfants face aux injustices sociales est un comportement appris des individus normalement responsables de promouvoir l'inclusion et la valorisation de la diversité sexuelle. Par conséquent, l'inconfort libère la parole queer et la légitime dans le contexte d'une discussion qui ouvre vers une critique des systèmes normatifs et normalisateurs qui dévalorisent le queer et, de manière plus générale, toute identité qui ne se plie pas et ne se conforme pas aux normes. Il y a donc une force de transformation qui émerge des œuvres queer.

Comme la revue $k w i R$ sert à engager le corps étudiant à prendre conscience de la diversité sexuelle et des injustices sociales qui y sont liées, elle comporte une dimension politique en contribuant à légitimer dans l'espace scolaire les modes de vie, les subjectivités et les expériences 
queer. Cette revue s'inscrit également dans une pédagogie queer qui serait éthiquement nécessaire pour l'ensemble des élèves, des enseignants et tout autre acteur dans le système éducatif. La construction d'une identité sexuelle saine est indispensable à la santé mentale de tout individu et, donc, la pédagogie queer aurait des bénéfices non seulement pour les élèves LGBTQ2+ dans la salle de classe, mais pour tout autre élève qui tente de construire son identité sexuelle et genrée librement.

\section{Conclusion}

Notre analyse avait pour but de montrer qu'à l'intérieur d'un système de pouvoir normalisateur comme l'école, la seule et l'unique façon de bouleverser l'ordre hétéronormatif est à travers le conflit. Le conflit dans ce contexte est lié à tout ce qui remet en cause l'hétérosexualité comme position sociale naturelle, voire tout ce qui rend plus visible et légitime la diversité sexuelle, la diversité d'identité de genre et la diversité d'expression de genre au sein des établissements scolaires. Il est néanmoins important de préciser que ce conflit est seulement productif lorsqu'il est déployé dans les espaces délimités par l'hétéronormativité et non dans les périphéries de celle-ci. Autrement dit, les réalités, expériences, modes de vie et subjectivités queer ne doivent pas être limités à des espaces privés qui l'empêchent de défier le climat hétéronormatif de l'école - pensons aux «GSA » ou «Gay-Straight Alliance ${ }^{2}$ - mais doivent être intégrés de manière transversale dans la salle de classe, dans le curriculum, dans les pratiques pédagogiques, dans la communauté scolaire, voire dans tous les espaces matériels et discursifs de l'école. C'est notamment ce que proposent Sean Currie, Maralee Mayberry et Tiffany Chenneville dans leur étude Destabilizing Anti-Gay Environments through Gay-Straight Alliances : Possibilities and Limitations through Shifting Discourses (2012). Si nous voulons que la diversité sexuelle et de genre soit réellement valorisée et que l'homophobie et la transphobie diminuent, il est alors impératif de bouleverser et de déstabiliser les «processus scolaires hétéronormalisateurs » (Currie et al., 60) qui contribuent à la ghettoïsation des identités et des réalités queer. Le fait d'éviter l'inconfort et le conflit serait donc une manière de contourner la résistance parentale ou communautaire, ce qui renforce l'homophobie et la transphobie en légitimant l'interdit autour de la diversité sexuelle et des expériences LGBTQ2+. Si la pédagogie queer privilégie le conflit dans le curriculum, elle est émancipatrice et profondément ancrée dans la justice sociale ; elle permet une critique de soi, de sa sexualité et de son genre. Pour ces raisons, la pédagogie queer est un 
vecteur important dans la construction du savoir, l'échange, le questionnement, la réflexion critique et surtout le changement paradigmatique dans l'organisation du genre et de la sexualité au sein de tout l'écosystème scolaire.

\section{Bibliographie}

Boulerice, Simon. L'enfant mascara. Montréal : Leméac, 2016.

Currie, Sean, et al. «Destabilizing Anti-Gay Environments through Gay-Straight Alliances : Possibilities and Limitations through Shifting Discourses ». The Clearing House 85.2 (2012) : 56-60.

Foucault, Michel. Surveiller et punir : Naissance de la prison. Paris : Gallimard, 1975.

---. Histoire de la sexualité I : La volonté de savoir. Paris : Gallimard, 1976.

Kjaran, Jón Ingvar. Constructing Sexualities and Gendered Bodies in School Space : Nordic Insights on Queer and Transgender Students. New York, NY : Palgrave Macmillan, 2017.

Mouffe, Chantal. L'illusion du consensus. Paris : Albin Michel, 2016.

Sedgwick, Eve Kosofsky. Epistemology of the Closet. Berkeley : U of California P, 1990.

Warner, Michael. The Trouble with Normal : Sex, Politics, and the Ethics of Queer Life. Cambridge : Harvard UP, 1999.

---. Publics and Counterpublics. New York : Zone, 2005.

\footnotetext{
Notes

${ }^{1}$ Pour accéder à la revue $k w i R$ dans son intégralité : https://larevuekwir.weebly.com

${ }^{2}$ Un «GSA » est une organisation étudiante normalement régie par un.e enseignant.e qui permet aux élèves LGBTQ2+ de se réunir dans un espace sécuritaire («safe space ») de l'école où ils peuvent former un réseau de soutien.
} 